ISSN 0103-5150

Fisioter. Mov., Curitiba, v. 25, n. 4, p. 701-707, out./dez. 2012

Licenciado sob uma Licença Creative Commons

\title{
A importância da análise dos gêneros para fisioterapeutas: enfoque nas quedas entre idosos
}

\author{
The importance of gender analysis to physical \\ therapists: focus on aging individuals falls
}

\section{Lívia Pimenta Renó Gasparotto ${ }^{[a]}$, José Francisco Fernandes Quirino dos Santos ${ }^{[b]}$}

[a] Mestre em Saúde Coletiva pela Universidade Federal de São Paulo (Unifesp), São Paulo, SP - Brasil, e-mail: livpi@hotmail.com

[b] Pós-doutor em Saúde Coletiva pela University of Toronto, doutor em Ciência Social, Antropologia Social pela Universidade de São Paulo (USP), professor convidado na linha de pesquisa Ciências Humanas em Saúde, da Pós-Graduação em Saúde Coletiva, Universidade Federal de São Paulo (Unifesp), São Paulo, SP - Brasil, e-mail: jquirino@uol.com.br.

\section{Resumo}

Introdução: 0 alto número de quedas entre pessoas idosas aumenta a demanda para reabilitação das debilidades físicas consequentes do evento, em clínicas de fisioterapia. Para evitar episódios recorrentes, o profissional dessa área deve alertá-los para práticas preventivas. Porém, trata-se de pessoas com diferentes contextos histórico-culturais e esquemas de rotina. Objetivo: Apontar as distinções entre gêneros como estratégia para a compreensão do cotidiano programado desses indivíduos. Materiais e métodos: Análise qualitativa, utilizando entrevista semiestruturada, partindo do tema da influência da queda na vida diária. As falas são transcritas codificadas e, posteriormente, categorizadas expressando as ideias encontradas no pensamento do idoso. Resultados: A queda doméstica está inserida no campo de trabalho da mulher (o lar) e, por isso, gera preocupação à medida que interfere nos hábitos do cotidiano referentes àquilo que define sua função no espaço e na vida. 0 homem parece preocupar-se com quedas quando ela impossibilita-o de manter um vínculo com o ambiente externo, o seu ambiente. Conclusão: Dessa forma, percebe-se que o gênero é uma categoria operacional em que se definem, para cada um, prioridades diferentes. Por meio de tal olhar, consegue-se justificar e compreender algumas relações de dominação existentes no seio da sociedade 
e que, ao fisioterapeuta, contribuem para que sejam discernidas certas particularidades entre pacientes que interferem na adesão a seus aconselhamentos preventivos.

Palavras-chave: Gênero. Envelhecimento. Fisioterapia. Quedas.

\section{Abstract}

Introduction: High rates of falls by elder people inevitably raise the demand for physical rehab in clinics. To efficiently cope with this, professional therapists should alert patients to preventive measures. However, they are people with different cultural and historical backgrounds and routines. Objective: To point out gender distinctions as a strategy for understanding these individuals' daily lives. Materials and methods: Qualitative data gathering and analysis, from semi-structured interviews, looking for any hidden meaning of falls in daily life at home. Opinions were taken and eventually ran into categories as to reveal the elders' own ideas. Results: The domestic fall is understood by women as a bad happening, right on their work space (home), thus raising a high level of concern, as it directly interferes with habits that are feminine and exclusive. To women, the fall jeopardizes their inner domain. Men, however, seem not to worry about falls, except when it prevents them from keeping linked to their proper masculine domain (the external milieu). Conclusion: By this approach, some real power relations can be outlined and understood. To the therapist, it helps to single out certain particulars that interfere with patients' prevention compliance pattern.

Keywords: Gender. Aging. Physical therapy. Falls.

\section{Introdução}

Estudos nas áreas de demografia, geriatria e gerontologia alertam sobre o aumento da população de idosos no país. Tal perspectiva aponta que, em menos de 20 anos, o Brasil estará entre as nações mais populosas de indivíduos idosos. Não obstante, pesquisas e políticas públicas investigam métodos para proporcionar a esse público (que cresce em ritmo comparativamente rápido) uma condição de vida melhor. Com isso, uma das expectativas é que sejam enfatizados, cada vez mais, tratamentos visando à manutenção da capacidade funcional dos indivíduos $(1,2,3)$.

Envelhecer é o processo de perda física e mental que acontece com todos os indivíduos não atingidos por doenças letais. Ao chegar à terceira idade, indivíduos deparam-se com decréscimos de massa óssea, muscular, modificações na homeostase circulatória, sem contar o envelhecimento do sistema nervoso, que altera não somente o aspecto físico mas também os de ordem comportamental, nem a autoconsciência de seu lugar perdido na sociedade. Sabe-se que certos cuidados preventivos realizados na fase jovem e adulta contribuem para que a fase do envelhecimento seja possivelmente vivida com saúde e autonomia física. Essas medidas promovem um tardio acometimento dos sintomas de fragilidade e é por isso que tanto se fala sobre atividade física para um envelhecimento saudável e prolongado $(4,5,6)$.

A queda na população idosa é fato de enorme preocupação em termos de saúde pública. Aponta-se que cerca de $30 \%$ dos idosos caem durante um ano. Somente esse dado já mostra gravidade, se considerarmos o tamanho do contingente populacional dessa faixa etária. Ainda assim, observa-se que, desses, 10\% voltam a cair e $5 \%$ fazem fratura de fêmur, que promove altos níveis de dependência física e, em casos mais graves, de restrição ao leito, frequentemente levam a óbito. Tratar da queda é, portanto, um assunto emergente entre profissionais de saúde, principalmente aqueles que trabalham com reabilitação $(7,8,9)$.

0 tratamento fisioterapêutico permite um contato próximo entre profissional e paciente, pois habitualmente são realizadas várias sessões entre eles, quando certos procedimentos são aplicados até atingir-se a recuperação física. No entanto, ao se pensar em quedas de idosos, esses mesmos indivíduos já reabilitados voltam para casa e continuam a se expor aos riscos domésticos que, em geral, são imperceptíveis aos olhos de quem habita aquele lar há tantos anos. Contudo, basta uma desatenção, ou, num plano mais sério, um pequeno rebaixamento da percepção de perigo, para que se produza a queda, ou as condições para que ela aconteça. Assim, o efetivo tratamento 
deve basear-se não somente no prontuário de técnicas aplicáveis à reabilitação, mas numa adequada informação a esses indivíduos para o conhecimento dos riscos e cuidados preventivos após o término da fisioterapia $(10,11,12)$.

Atualmente, o acúmulo de informação técnica sobre doença e tratamentos faz com que o profissional se sinta seguro quanto à efetiva recuperação física de sua clientela. Além disso, certas informações são menos comentadas entre especialistas e participam de uma parte imprescindível do tratamento: a adesão aos cuidados indicados pelo profissional, fora da clínica de reabilitação. Por exemplo, o presente estudo fez uma análise dos aspectos ligados a gênero e sua importância no processo de envelhecimento, assim como das providências tomadas pelo(a) idoso(a) $(13,14,15)$.

0 termo gênero é muito útil para caracterizar o indivíduo segundo suas diferentes percepções e condutas de vida, influenciadas pelo contexto histórico do homem e da mulher em sua posição e papéis na sociedade. A palavra gênero sofre certa distorção etimológica pela sociedade (inclusive por pesquisadores), uma vez que é facilmente dita para referenciar o sexo (masculino ou feminino). Gênero, ao contrário de sexo, não diferencia o indivíduo por características biológicas, mas pauta-se pela determinação histórica e comportamental construída por homens e mulheres. Ao fisioterapeuta é importante compreender esses aspectos no momento de expor ao indivíduo idoso a necessidade de condutas preventivas. A necessidade de mudar de padrões de vida no lar, para que sejam inteligíveis por eles(as), precisam adequar-se a suas concepções e seus costumes de vida, ou pelo menos estar próximas disso. Essa é uma condição de eficiência para tratamentos em geral $(16,17)$.

\section{Materiais e métodos}

0 estudo foi realizado entre outubro e novembro de 2009, com opiniões de 15 idosos moradores de um bairro paulistano de classe média baixa. Os indivíduos participam do projeto EPIDOSO, do Centro de Estudos do Envelhecimento da Unifesp, projeto que acompanha uma coorte de idosos analisando dados geriátricos e gerontológicos há mais de dez anos. 0 trabalho passou pela aprovação do comitê de ética e pesquisa da universidade, sob o parecer n. 0429/09. Para fins legais, a pesquisa contou com a utilização do Termo de Consentimento Livre e Esclarecido (TCLE) pelos participantes.

O objetivo da pesquisa foi obter concepções de pessoas idosas sobre o evento da queda. Para tanto, optou-se pela metodologia de pesquisa qualitativa em razão do direcionamento desta na análise de concepções pela perspectiva do entrevistado.

Com isso, inicialmente definiu-se como participantes da pesquisa pessoas idosas com idade acima de 60 anos e que tivessem sofrido uma ou mais quedas no ano corrido. Foram descartados aqueles que, por conta da queda, tiveram perda da autonomia das atividades de vida diária (AVDs). Também foram excluídos do estudo aqueles com pouca acuidade visual, pouca capacidade de raciocínio lógico e deficit auditivo. Isso porque, para obter as dados por meio de entrevistas, é necessária a participação ativa do idoso.

Por se tratar de um tema relativo ao ambiente doméstico, definiu-se que a pesquisa seria realizada nesse local, a fim de enriquecer a análise por ser possível ilustrar o relato com amostragem dos fatos. 0 espaço também promove maior comodidade e familiaridade para o entrevistado, predispondo-o a se sentir confortável diante da atividade proposta, 0 que contribui para aumento de sua espontaneidade.

0 estudo qualitativo parte de uma análise do conhecimento do indivíduo sobre um assunto previamente determinado. Para isso, a pesquisa foi realizada por meio de entrevista semiestruturada aberta, em que as questões relativas ao tema central são sugeridas ao idoso, estimulando-o a falar sobre elas a partir de seus conceitos. A espontaneidade do indivíduo torna a análise mais coerente com sua forma de raciocínio e mediação de ideias. 0 participante foi conduzido a contar suas rotinas, suas definições de tarefas e detalhes de seu cotidiano considerados importantes. Após esse exercício narrativo, a atenção foi dirigida para a queda ocorrida naquele cenário doméstico e as possíveis interferências desse episódio no seu dia a dia (18).

As entrevistas foram gravadas e, posteriormente, transcritas e codificadas pela técnica axial e seletiva. Segundo essa metodologia, as ideias recorrentes entre os participantes, isto é, falas que mostram em todos os participantes uma similaridade de ideias são identificadas e agrupadas em grupos de pensamentos. Em seguida, esses grupos são categorizados e, com isso, as classes de pensamento sobre o tema proposto são descobertas e relatadas $(19,20)$. 
Mais adiante, será discutida a categorização relativa ao gênero, no contexto da queda doméstica.

\section{Resultados e discussão}

Dos 15 participantes, 4 homens e 11 mulheres, verificou-se a média de idade de 74,8 anos. Esses idosos participam do estilo de família multigeracional, encontrada comumente em residências brasileiras, na qual os moradores são avôs, filhos, netos e ou agregados. No caso da pesquisa, 11 deles moravam com netos, filhos ou cuidadores, e apenas 4 habitavam com o cônjuge. Todos os homens entrevistados moravam com as esposas. Apenas três entrevistados moravam em apartamentos.

\section{Análise do ambiente}

Por se tratar de um bairro de classe média baixa, foram observados muitos cômodos no ambiente doméstico, grande parte deles com acúmulo de móveis antigos e novos, concentração de objetos sobre as cômodas, como porta-retratos, objetos antigos.

Como elemento de risco para quedas, notamos desníveis localizados na passagem de um cômodo ao outro, ou mesmo no quintal. Também foram encontrados tapetes capachos e passadeiras em salas e quartos, além da falta de adaptação ou suporte de segurança em banheiros, mobílias pontiagudas e de ergonomia desfavorável (ex.: camas muito baixas, que exigem maior esforço para levantar, e armários de cozinha em locais altos, exigindo a utilização de cadeira para acessar algum objeto). Essa realidade está presente em pesquisas que analisam aspectos relacionados ao risco ambiental para quedas domésticas nas casas de pessoas idosas $(21,22)$.

A seguir, serão mostradas as características de gênero referentes a certos detalhes da rotina desses idosos. Na análise de gênero, procuramos os papéis socialmente significativos desempenhados por homens e mulheres.

A rotina do lar e a influência da queda na perspectiva de gênero

Em ambientes domésticos de idosos e idosas, observou-se que a distribuição das tarefas de rotina se dá de forma característica, reproduzindo no lar as especificidades sociais de gênero vigentes na sociedade $(22,23)$. Exemplos do modo de vida característico da mulher foram retirados das falas:

Minhas prioridades sempre foram alimentação $e$ roupa em dia. Então, eu gosto de, pela manhã, fazer as minhas coisinhas tranquilamente, nem que seja meia hora (M 11, 64 anos).

Quem me vê sair de casa vê outra pessoa! Aqui eu sou a Neusa, dona de casa. Aqui fico mais à vontade com a roupa, com o sapato. Ponho o lencinho na cabeça, e essa sou eu! (M 12, 66 anos).

A identificação da casa como parte do trabalho diário, exercido por tarefas domésticas ou atenção dedicada ao ambiente, é observada em mulheres idosas dessa realidade urbana, mas também em outras culturas, por exemplo, as tribos indígenas. Nessa última, mulheres têm a tarefa do cuidado dos filhos, dos alimentos e da casa. A fase da terceira idade induz à mudança de rotina, já que sua maioria populacional apresenta-se aposentada. Dessa forma, no que diz respeito às idosas, o lar passa a ser o espaço de maior acesso diário e, portanto, é nele que se determinam e reproduzem as principais funções diárias $(24,25)$.

Ainda nesse cenário, a preocupação com os familiares próximos - já vista também nas comunidades indígenas - ocorre de forma semelhante. As falas seguintes retratam a dedicação da mulher idosa em relação ao marido e seus descendentes.

Depois que meu marido toma banho, eu tomo o meu ou, então, deixo caso ele queira que eu o ajude em alguma coisa, porque ele me chama o dia inteirinho! (M 6, 80 anos).

Então tudo me dói. Se eu fico nervosa então... Eu tenho dores horríveis. Daí não dá pra fazer nada. Eu só não deixo faltar alimentação para meu marido e meu neto (M 7, 72 anos).

A divisão do trabalho especializa a mulher nas tarefas domésticas, transformando o lar no seu local de trabalho, ao contrário do homem, que comumente trabalha fora de casa. Essa distinção é crucial durante a velhice, pois o homem, aposentado, passa do ambiente de trabalho para a maior permanência 
no lar - uma quebra fundamental de sua rotina. Já a mulher, ainda que se aposente, permanece em seus domínios domésticos quando envelhece, tendo, por isso, uma mudança menor no seu cotidiano. Essa especialização de gênero, típica, tem consequências na consciência de si dos idosos; o homem vê-se confinado em um espaço que para ele, claramente, não é o seu e no qual se move com menos fluência. Já a mulher idosa move-se no lar como sempre fez $(24,26)$.

Uma segunda distinção estaria na maior familiaridade da mulher com os serviços de saúde em geral, como consumidora frequente desses serviços. 0 homem, de seu lado, aderente aos cânones do comportamento masculino, pouco se queixa, pouco vai ao médico, pouco percebe da sua condição de saúde. Não é um frequentador de salas de espera de clínicas $(23,27)$.

Entretanto, nos dois parágrafos anteriores, há muito de estereótipos e pouco de pesquisa de campo. Contudo, existem dados suficientes no trabalho, aqui resumidos, a fim de confirmar que a mulher "vive" mais que o homem, uma vez que ela se trata e adere a tratamentos mais facilmente. Portanto, a queda doméstica se insere no campo de trabalho da mulher, porém inação do homem. Obviamente, há consequências disso, importantes na consciência de si de cada um $(28,29)$.

A queda, um evento simples em si, possui componentes fisiológicos, psíquicos e sociais de complexidade. Como todo fato de significação social, afeta diferentes percepções segundo o contexto social e histórico em que se inscreva (30). Ao analisar o comportamento e percepção de homens e mulheres nessa faixa etária, notam-se duas conduções de ideias distintas sobre essa temática.

Para algumas mulheres, dada a intensa e permanente relação com o ambiente doméstico, a queda gera preocupação à medida que interfere nesses hábitos do cotidiano referentes àquilo que define sua função no espaço e na vida. A possibilidade de realizar as tarefas domésticas é, portanto, ferramenta que define a idosa como mulher ativa, útil, dando-lhe, por isso, uma percepção positiva sobre envelhecimento. A independência desempenha, nesse caso, um papel crucial e único (16).

Quando estava boa, eu fazia tudo porque eu sou enjoada. Agora a casa está deixada, coitada. Eu gosto de tudo bem limpinho, tiro o pó todo o dia, a gente lava uma coisa e lava outra (M 9, 84 anos).
O pior é a dor nas pernas. Já não dá para andar muito como antes, varrer a casa já não posso (M 15, 70 anos).

Assim, impedir a mulher de realizar tais tarefas é, ainda que se reduza o risco para quedas, impedi-la de conduzir um modo de vida que lhe dá sentido. Sendo assim, suspender essas atividades não parece a melhor conduta a ser indicada pelo profissional fisioterapeuta. Nesse aspecto, vale lembrar que há formas de readaptar a mesma atividade considerando os aspectos biomecânicos e de força física do corpo, para que o exercício seja desempenhado de maneira segura $(13,22)$.

No caso do homem, a preocupação com quedas refere à impossibilidade de manter um vínculo com o ambiente externo com o qual ele tem maior afinidade. Na idade avançada, o homem, na maioria das vezes aposentado, passa a maior parte do tempo em casa, mas procura de algum modo tomar para si atividades que reconstroem uma relação com outros espaços, adquirida anteriormente em seu trabalho. Tarefas como pagar uma conta no banco, ir à farmácia, comprar o jornal, levar alguém ao mercado permitem uma responsabilidade não relativa ao lar, onde o domínio do espaço e da situação pertence à mulher $(4,15,27)$.

Eu acordo, dou uma lida rápida no jornal, depois eu vejo no escritório essas coisas de pagar, se tem que pagar alguma coisa de banco, essas coisas, se tem alguma intervenção a fazer em banco (H 5 , 80 anos).

Como sugestão, o trabalho aponta formas de realizar os aconselhamentos preventivos de queda considerando a relação distinta no modo de vida de homens e mulheres idosos. Assim, aconselhar um homem idoso sobre riscos de queda é lembrar que, para ele, atividades externas têm importante significado na definição de sua rotina. Uma alternativa para que ocorra o aprendizado sobre qualquer recomendação de prevenção de riscos é alertá-lo a respeito da necessidade de cuidar da saúde física do corpo com a meta de dar continuidade a um cotidiano ativo dotado da relação permanente com outros espaços. Já no caso da idosa, o foco deve ser a importância da saúde funcional como promotora da autonomia para atividades diárias, permitindo um cotidiano ativo no lar.

Dessa forma, percebe-se que o gênero é uma categoria operacional em que se definem, para cada um, prioridades diferentes $(16,17)$. Assim, consegue-se 
justificar e compreender algumas relações de dominação existentes no seio da sociedade e que, ao fisioterapeuta, contribuem para que sejam discernidas certas particularidades entre pacientes que interferem na adesão a seus aconselhamentos preventivos.

Algumas atitudes devem ser tomadas na definição de condutas preventivas para homens e mulheres idosos e é preciso partir da retomada de tais rotinas. Essas informações podem ser adquiridas no contato do fisioterapeuta com o seu paciente durante o tratamento. Assim, antes de indicar meios de prevenção para quedas em casa, o profissional tem de demonstrar interesse pela forma como o idoso elabora sua rotina e entender, pela contribuição do estudo de gênero, as distintas atitudes que influenciam tais condutas, isto é, deve inscrever a queda no contexto específico de cada paciente.

\section{Considerações finais}

0 tratamento fisioterapêutico para quedas não deve dar-se por terminado ao final das sessões. É importante que o profissional identifique atividades do cotidiano dos idosos que lhes dão sentido à rotina e se baseie nelas para indicar adequadamente as maneiras de se prevenir de novas quedas no espaço do lar.

A convivência entre paciente e profissional durante as sessões oferece oportunidade de tempo para que tais informações sejam absorvidas e inseridas na maneira de indicar as condutas preventivas necessárias. 0 próprio paciente fornece naturalmente as categorias mais inteligíveis para ele, facilitando o lado da comunicação desejada pelo fisioterapeuta para ampliar sua eficiência.

\section{Agradecimento}

Agradecemos à Capes pelo incentivo financeiro.

\section{Referências}

1. Maia FOM, Duarte YAO, Lebrão ML, Santos JLF. Risk factors for mortality among elderly peoples. Rev Saude Publica. 2006;40(6)745-53.
2. Ramos LR. Fatores determinantes para o envelhecimento saudável em idosos residentes em centro urbano: Projeto Epidoso, São Paulo. Cad Saúde Pública. 2003;19(3):7938. doi:10.1590/S0102-311X2003000300011.

3. Instituto Brasileiro de Geografia e Estatística - IBGE. Indicadores sociodemográficos e de saúde no Brasil. [citado 14 jun. 2010]. Disponível em: http://www. ibge.gov.br/home/estatistica/populacao/indic_sociosaude/2009/indicsaude.pdf.

4. Murtag KN, Hubert HB. Gender differences in physical disability among an elderly cohort. Am J Public Health. 2004;94(8):1406-11. doi:10.2105/AJPH.94.8.1406.

5. Alley D, Sutheres K, Crimmins E. Education and cognitive decline in older Americans. Res Aging. 2007; 29(1):73-94. doi:10.1177/0164027506294245.

6. Abreu DC. The association between osteoporosis and static balance in elderly women. Osteoporos Int. 2011;21(9):1487-91. doi:10.1007/s00198-009-1117-5.

7. Liu-ambrose TY, Ashe MC, Graf P, Beattie B, Khan KM. Increased risk of falling in older community-dwelling woman with mild cognitive impairment. Phys Ther. 2008;88(12):1482-91. doi:10.2522/ptj.20080117.

8. Pinheiro MM, Ciconelli RN, Martini LA, Ferraz MB. Risk factors for recurrent falls among Brazilian women and men: the Brazilian Osteoporozys study (BRAZOS). Cad Saude Publica. 2010;26(1):89-96. doi:10.1590/ S0102-311X2010000100010.

9. Masud T, Morris RO. Epidemiology of falls. Age Ageing. 2001;30(s4):3-7. doi:10.1093/ageing/30.suppl_4.3.

10. Stoddart H, Sharp D, Harvey I, Whitley H. Falls and the use of services in community-living elderly people. Br J Gen Pract. 2002;52:923-25. PMid:12434962.

11. Wray LA, Blaum CS. Explaining the role of sex on disability: a population-based study. Gerontologist. 2001; (41):499-510. doi:10.1093/geront/41.4.499.

12. Mossey JM, Shapiro E. Self-rated health: a predictor of mortality among the elderly. Am J Public Health. 1992; 72(8):800-8. doi:10.2105/AJPH.72.8.800.

13. American Geriatrics Society, British Geriatrics Society, American Academy of Orthopaedic Surgeons Panel on falls preventions. Guideline for the preventions falls in older persons. J Am Geriatr Soc. 2001;49(5):664-672. doi:10.1046/j.1532-5415.2001.49115.x. 
14. Fabrício SCC, Rodrigues RAP, Costa Júnior ML. Causas e consequências de quedas de idosos atendidos em hospital público. Rev Saúde Pública. 2004;38(1):93-9. doi:10.1590/S0034-89102004000100013.

15. Jardim VCFS, Medeiros BF, Brito AM. Um olhar sobre o processo do envelhecimento: a percepção de idosos sobre a velhice. Rev Bras Geriatr Gerontol. 2006; $9(2): 25-34$.

16. Fonseca RM. Equidade de gênero e saúde das mulheres. Rev Esc Enferm USP. 2005;39(4):450-459. doi:10.1590/S0080-62342005000400012.

17. Martin D, Andreolli SP, Quirino J, Nakamura E. Noção dos significados nas pesquisas qualitativas em saúde: a contribuição da antropologia. Rev Saúde Pública. 2006,40(1):178-9. doi:10.1590/ S0034-89102006000100026.

18. Camargo Junior KR. A relevância sobre o uso de técnicas qualitativas em pesquisas sobre a biomedicina. Cien Saude Colet. 2008;13(4):1327-30. doi:10.1590/ S1413-81232008000400029.

19. Flick U. Introdução à pesquisa qualitativa. 3. ed. São Paulo: Artmed, 2009.

20. Willms DG, Johnson NA. Essentials in qualitative research: a notebook for the field. 1993 [mimeo].

21. Salvador EP, Florindo AP, Reis RS, Costa EF. Percepção do ambiente e prática de atividade física no lazer entre idosos. Rev Saude Publica. 2009;43(6):972-80. doi:10.1590/S0034-89102009005000082.

22. Fabrício SSC, Rodrigues RAP. Percepção de idosos sobre alterações das atividades da vida diária após acidente por queda. Rev Enferm UERJ. 2006;14(4):531-7.

23. Doimo LA, Derntl AM, Lago OC. O uso do tempo do cotidiano de mulheres idosas: um método indicador do estilo de vida de grupos populacionais. Cien Saude Colet. 2008;(13)4:1133-42. doi:10.1590/ S1413-81232008000400009.
24. Clastres, P. $O$ arco e o cesto. In Clastres, P. (editor). A sociedade contra o Estado: pesquisas de antropologia política. Rio de Janeiro: Francisco Alves, 1978.

25. Beauvoir S. Les structures élémentaire de la parenté, Claude Lévi-Strauss. Les Temps Modernes. 1949; 7(49):943-9.

26. Barreto SM, Figueiredo RC. Doença crônica, auto-avaliação de saúde e comportamento de risco: diferença de gênero. Rev Saude Publica. 2009;43(supl.2):38-47.

27. Coutinho RX, Acosta MAF. Ambientes masculinos da terceira idade. Cien Saude Colet. 2009;14(4):1111-18. doi:10.1590/S1413-81232009000400017.

28. Menec V. The relation between everyday activities and succesfull aging: a 6 year longitudinal study. J Gerontol B Psychol Sci Soc Sci. 2003,58(2):S74-82.

29. Canesqui AM. Olhares socioantropológicos sobre os adoecidos crônicos. São Paulo: Hucitec; FAPESP, 2007.

30. Morris JÁ, Mackenzie EJ, Eldestein SL. The effect of preexisting conditions on mortality in trauma patients. JAMA. 1990;(263):1942-6. doi:10.1001/ jama.1990.03440140068033.

Recebido: 09/08/2011

Received: 08/09/2011

Aprovado: $23 / 04 / 2012$

Approved: 04/23/2012 